\title{
SELF-ADJOINT OPERATORS AND THE GENERAL GKN-EM THEOREM
}

\author{
LANCE L. LiTTLEJOHN AND RICHARD WELLMAN
}

\begin{abstract}
We construct self-adjoint operators in the direct sum of a complex Hilbert space $H$ and a finite dimensional complex inner product space $W$. The operator theory developed in this paper for the Hilbert space $H \oplus W$ is originally motivated by some fourth-order differential operators, studied by Everitt and others, having orthogonal polynomial eigenfunctions. Generated by a closed symmetric operator $T_{0}$ in $H$ with equal and finite deficiency indices and its adjoint $T_{1}$, we define families of minimal operators $\left\{\widehat{T}_{0}\right\}$ and maximal operators $\left\{\widehat{T}_{1}\right\}$ in the extended space $H \oplus W$ and establish, using a recent theory of complex symplectic geometry, developed by Everitt and Markus, a characterization of self-adjoint extensions of $\left\{\widehat{T}_{0}\right\}$ when the dimension of the extension space $W$ is not greater than the deficiency index of $T_{0}$. A generalization of the classical Glazman-Krein-Naimark (GKN) theorem - called the GKN-EM theorem to acknowledge the work of Everitt and Markus - is key to finding these self-adjoint extensions in $H \oplus W$. We consider several examples to illustrate our results.
\end{abstract}

Mathematics subject classification (2010): 05C38, 15A15, 05A15, 15A18.

Keywords and phrases: Symmetric operator, self-adjoint operator, differential operator, maximal operator, minimal operator, Glazman-Krein-Naimark theory, symplectic GKN theorem, orthogonal polynomials.

\section{REFERENCES}

[1] N. I. AKhiEZER And I. M. GlaZman, Theory of Linear Operators in Hilbert space, Parts I and II, Scottish Academic Press, Pitman Advanced Publishing Program, London, U.K. 1981.

[2] N. Dunford And J. T. Schwartz, Linear Operators, Part II, John Wiley Publishers, New York, 1963.

[3] W. N. Everitt, A. M. Krall, And L. L. Littlejohn, On some properties of the Legendre type differential expression, Quaestiones Math., 13(1), 1990, 83-116.

[4] W. N. EVERITT AND L. L. LitTLEJohn, Differential operators and the Legendre type polynomials, Differential and Integral Equations, 1(1), 1988, 97-116.

[5] W. N. Everitt And L. L. LitTlejohn, Orthogonal polynomials and spectral theory: a survey, Proceedings of the III International Symposium on Orthogonal Polynomials and Applications, Erice, Italy, 1990. IMACS Annals on Computing and Applied Mathematics 9(1991), 21-55; J. C. Baltzer AG, Basel, Switzerland, 1991, 21-55.

[6] W. N. Everitt, K. H. Kwon, L. L. Littlejohn And R. Wellman, Orthogonal polynomial solutions of linear ordinary differential equations, Proceedings of the Fifth International Symposium on Orthogonal Polynomials, Special Functions and their Applications (Patras, 1999), J. Comput. Appl. Math. 133 (2001), no. 1-2, 85-109.

[7] W. N. EveritT AND L. MARKus, The Glazman-Krein-Naimark Theorem for Ordinary Differential Operators, Operator Theory: Advances and Applications 98(1997), 118-130.

[8] W. N. Everitt And L. Markus, Complex Symplectic Geometry with Applications to Ordinary Differential Operators, Trans. Amer. Math. Soc., 351(12), 1999, 4905-4945.

[9] W. N. Everitt And L. Markus, Boundary Value Problems and Symplectic Algebra for Ordinary Differential and Quasi-Differential Operators, Math. Surveys Monogr., Volume 61, American Mathematical Society, Providence, Rhode Island, 1999.

[10] W. N. Everitt And L. Markus, Complex Symplectic Spaces and Boundary Value Problems, Bull. Amer. Math. Soc. (N.S.) 42 (2005), no. 4, 461-500. 
[11] V. Guillemin And S. Sternberg, Symplectic techniques in physics, Cambridge University Press, 1990.

[12] A. M. Krall, On Orthogonal Polynomials Satisfying Fourth Order Differential Equations, Proc. Roy. Soc. Edinb. (A), 1981, 271-288.

[13] H. L. KRall, Certain Differential Equations for Tchebycheff polynomials, Duke Math J., 4. 1938, $705-718$.

[14] H. L. KRALL, Orthogonal polynomials satisfying a Certain fourth order differential equation, The Pennsylvania State College Studies, No. 6, The Pennsylvania State College, State College, PA. 1940.

[15] M. A. NAimark, Linear Differential Operators, Part II, Ungar Publishing Co., New York, 1968.

[16] M. Reed And B. Simon, Methods of Modern Mathematical Physics I. Functional Analysis, Academic Press, New York, 1972.

[17] W. Rudin, Functional Analysis (2nd edition), McGraw-Hill Publishers, New York (1991).

[18] M. Stone, Linear transformations in Hilbert space, American Mathematical Society Colloquium Publications 15, Providence, RI, 1932.

[19] J. Weidmann, Linear operators in Hilbert space, Springer-Verlag, Heidelberg, 1980.

[20] A. ZETTL, Formally self-adjoint quasi-differential operators, Rocky Mountain J. Math., 5(1975), 453474.

[21] A. ZetTL, Sturm-Liouville theory, Mathematical Surveys and Monographs, 121, American Mathematical Society, Providence, RI, 2005. 